Brit. Heart J., 1965, 27, 236.

\title{
VECTORCARDIOGRAPHY IN CONGENITAL HEART DISEASE WITH THE USE OF THE FRANK SYSTEM*
}

\author{
BY \\ ALBERTO BENCHIMOL AND EVANDRO G. LUCENA \\ From The Institute for Cardiopulmonary Diseases, Scripps Clinic and Research Foundation, La Jolla, California, U.S.A.
}

Received May 25, 1964

In the past few years vectorcardiography has gained wide acceptance as a diagnostic tool in cardiovascular diseases. Most of the present knowledge with this technique is based on vectorcardiograms recorded with the use of the cube system and the tetrahedron as proposed by Grishman, Borun, and Jaffe (1951), Scherlis, Lasser, and Grishman (1951), Duchosal and Sulzer (1949), Wilson and Johnston (1938), and Burch, Abildskov, and Cronvich (1953). More recent evidences indicate that the corrected orthogonal systems as described by Frank (1956), McFee and Johnston (1954), Schmitt and Simonson (1955), and others (Simonson, Schmitt, and Levine, 1955; Burger, Van Milaan, and Boer, 1952; Pipberger and Lillienfield, 1958) provide a more accurate description of the abnormalities of the electrical activation of the heart as compared with the non-corrected orthogonal systems. Furthermore, significant changes in morphology, contour, and spatial orientation of the QRS vectors are seen in loops recorded in the same patient using different systems (Langner et al., 1958; Pipberger, 1959) (Fig. 1). This report reviews our experience with a large number of vectorcardiograms recorded in patients with documented congenital heart disease.

\section{SubJECTS AND MeTHODS}

Our experience with vectorcardiograms (VCG) recorded with the Frank system is based on more than 2000 patients with various forms of heart diseases, throughout a period of three years. Approximately 200 of them had congenital heart disease of the cyanotic and acyanotic types. The vast majority had the diagnosis confirmed by cardiac catheterization, indicator-dilution technique, and cine-angiocardiography. Of this proved group, most had further surgical or necropsy confirmation of the anomalies. There were 100 patients with normal cardiovascular systems and they were used as a control group.

The VCGs were recorded using a Hart Electronics, Model PV3, vectorcardiographic machine. $\dagger$ The loops were photographed from the oscilloscope with a polaroid camera using a 3.000 speed polaroid land film (10 seconds developing time). The Frank electrode system was employed in all cases using the fifth intercostal space for placement of the præcordial electrodes. The VCGs were recorded at or near the same time as the electrocardiogram. All patients had still loops recorded in the frontal, left sagittal, and horizontal planes. In addition to the still loops, running loops at various speeds were recorded in order to provide a better analysis of the intimal and terminal components of the QRS vector. The loops were interrupted every two milliseconds (msec.) and 1 millivolt standardization was recorded with each loop. The amplitude, direction, magnitude, and rotation of the individual QRS vectors were noted in each case. The measurements made in 41 normal subjects are illustrated in the Table. The Helm system (Helm, 1956) was used to express the direction of the vectors.

* This study was supported in part by NIH Graduate Training Grant HTS-5513, NIH Research Grant HE-07983-01 and by the Timken-Sturgis Foundation.

$\dagger$ Hart Electronics, San Pedro, California. 


\section{RESUlTS AND COMMENTS}

\section{Normal Vectorcardiogram}

No attempt is made to describe in great detail the characteristics of a normal vectorcardiogram (VCG). Excellent reviews of the normal VCG can be found in earlier reports (Jouve et al., 1950; Hugenholtz and Liebman, 1962; McCall, Wallace, and Estes, 1962; Forkner, Hugenholtz, and Levine, 1961; Bristow, 1961). The measurements made in the normal VCG are illustrated in the Table.

Frontal plane: in this plane the rotation of the loop can be either clockwise or counterclockwise. The initial 10 and/or $20 \mathrm{msec}$. vectors are usually directed superiorly and to the right with a maximum deflection vector averaging 46 degrees (Fig. 2 and 3).

Sagittal plane: the rotation in this plane is always counterclockwise with the exception of infants and young children up to the age of 4 years in whom the rotation may be clockwise or in figure-of-eight in the absence of heart disease (Fig. 3). The initial component in this plane is usually directed anteriorly and superiorly with a maximum QRS vector at an average of 70 degrees.

Horizontal plane: the rotation is always
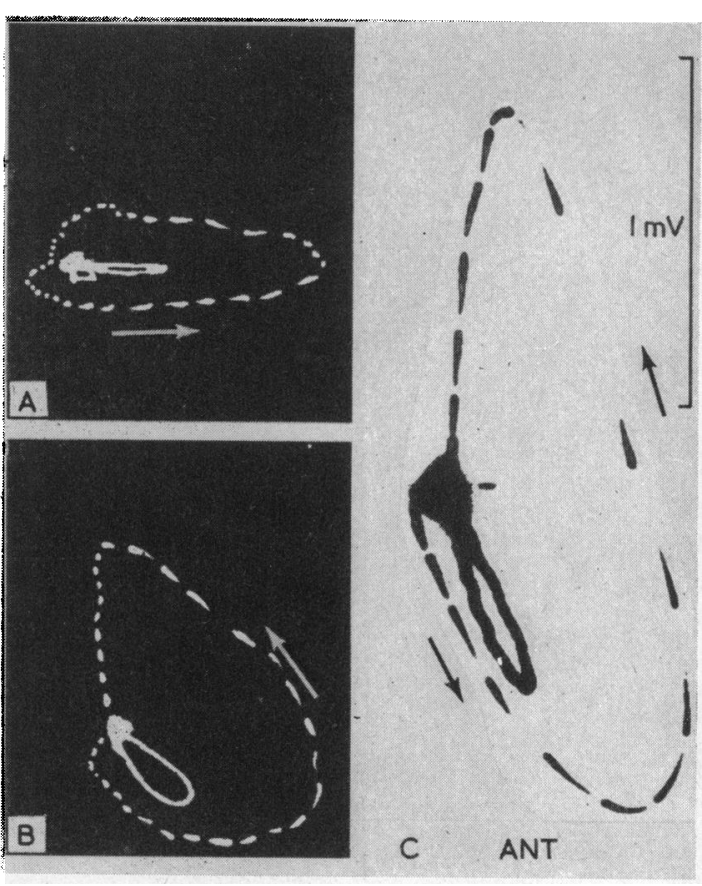

FIG. 1.-Normal subjects. Horizontal loops recorded in the same subject using the cube system (A), the Frank system (B), and the McFee-Johnston system (C). Note the narrow loop in A, the round loop in $B$, and the large anterior displacement in $C$ (see text). counterclockwise and the remarks relating to the sagittal plane also apply for the horizontal loop. The initial component representing septal depolarization is directed anteriorly and to the right and lasts 5 to $15 \mathrm{msec}$. The 20,30, 40, and $50 \mathrm{msec}$. vectors are inscribed in the posterior and left quadrants. The main body of the QRS loop is located in the posterior and left quadrant and the average maximum deflection vector is at 320 degrees.

Occasionally respiratory variations are recorded, being more pronounced in the frontal and sagittal loops. However, these variations are of a much lesser degree as compared with variations seen in the electrocardiograms. When they occur they are characterized by a shift in direction and/or rotation of the initial component in the frontal and sagittal planes as illustrated in Fig. 4.

TABLE

Average Measurements of the Various Vectors made in 41 Normal Vectorcardiograms Racorded With the. FRANK SYSTEM

\begin{tabular}{|c|c|c|c|c|c|c|c|c|c|c|}
\hline \multirow{2}{*}{ Plane } & & \multicolumn{5}{|c|}{ QRS loop-dir.-deg. } & \multicolumn{2}{|c|}{ QRS-MDV* } & \multirow{2}{*}{$\begin{array}{c}\text { P loop } \\
\begin{array}{l}\text { Dir. } \\
\text { deg. }\end{array}\end{array}$} & \multirow{2}{*}{$\begin{array}{l}\text { T loop } \\
\begin{array}{l}\text { Dir. } \\
\text { deg. }\end{array}\end{array}$} \\
\hline & & $\begin{array}{c}10 \\
\text { msec. }\end{array}$ & $\begin{array}{c}20 \\
\text { msec. }\end{array}$ & $\begin{array}{c}30 \\
\text { msec. }\end{array}$ & $\begin{array}{c}40 \\
\text { msec. }\end{array}$ & $\begin{array}{c}50 \\
\text { msec. }\end{array}$ & $\begin{array}{l}\text { Dir. } \\
\text { deg. }\end{array}$ & $\underset{\text { mv }}{\text { Mag. }}$ & & \\
\hline $\begin{array}{l}\text { Frontal.. } \\
\text { Sagittal. . } \\
\text { Horizontal }\end{array}$ & $\begin{array}{l}\cdots \\
\cdots \\
\cdots\end{array}$ & $\begin{array}{r}253 \\
186 \\
94\end{array}$ & $\begin{array}{r}39 \\
140 \\
45\end{array}$ & $\begin{array}{r}43 \\
88 \\
342\end{array}$ & $\begin{array}{r}59 \\
55 \\
313\end{array}$ & $\begin{array}{r}155 \\
39 \\
269\end{array}$ & $\begin{array}{r}46 \\
70 \\
320\end{array}$ & $\begin{array}{l}1 \cdot 64 \\
1 \cdot 27 \\
1 \cdot 34\end{array}$ & $\begin{array}{l}57 \\
88 \\
44\end{array}$ & $\begin{array}{r}50 \\
116 \\
40\end{array}$ \\
\hline
\end{tabular}

* Maximum deflection vector 

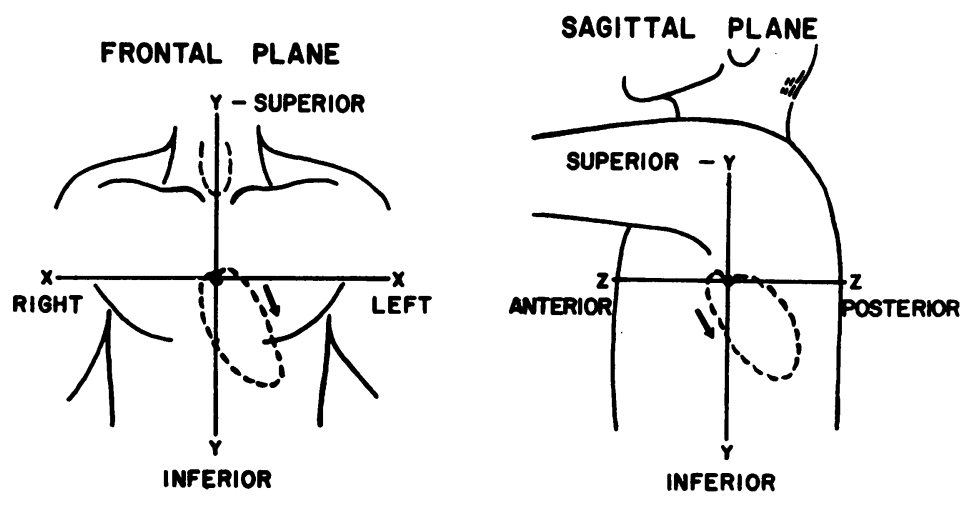

horizontal plane

POSTERIOR

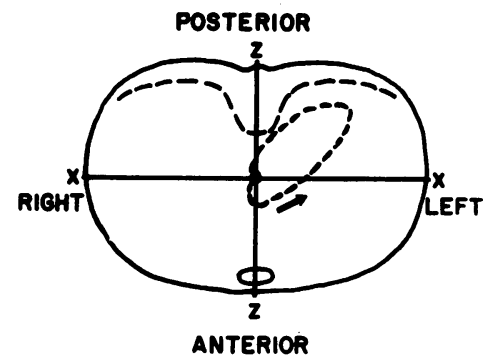

FIG. 2.-Diagram of a normal vectorcardiogram in the frontal, sagittal, and horizontal planes (see text):

Physiological changes recorded in the electrocardiogram occurring as a function of age have been described. However, this physiological right ventricular preponderance is not usually detected in the VCG recorded with the Frank system (Hugenholtz and Liebman, 1962).
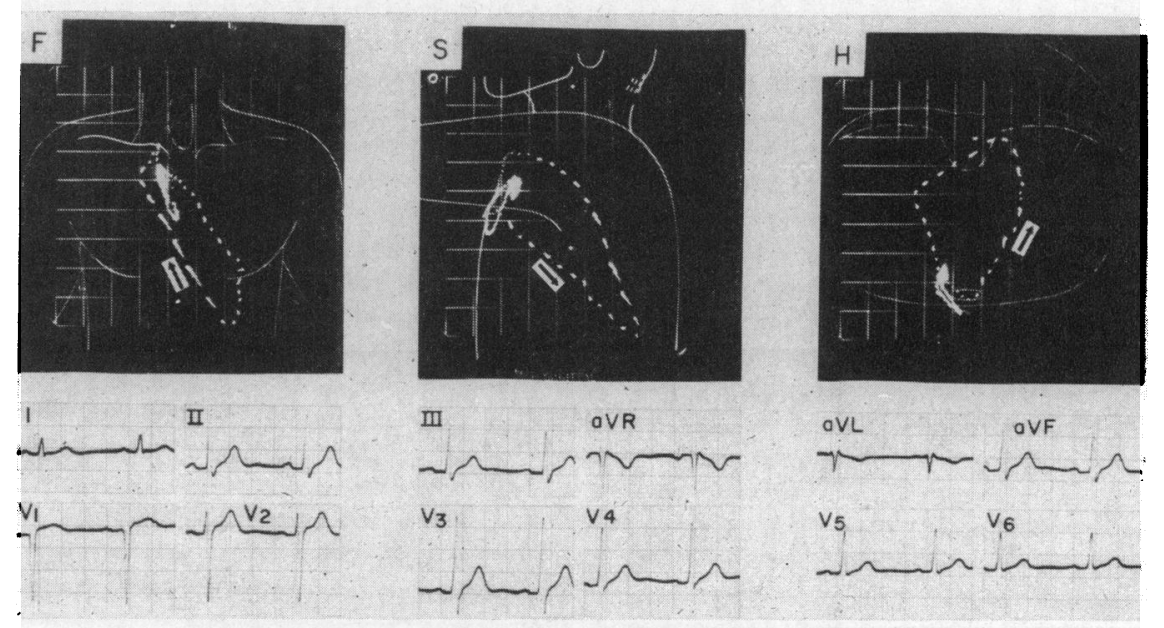

FIG. 3.-Normal vectorcardiogram in the frontal, sagittal, and horizontal planes (see text). 


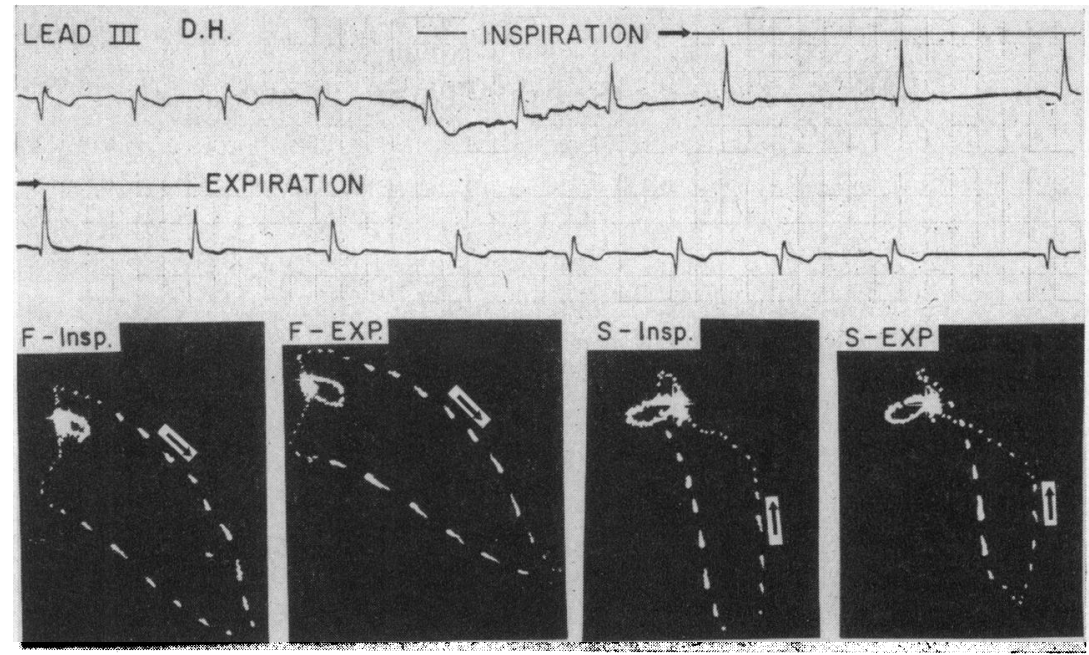

Fig. 5.-Atrial septal defect without pulmonary hypertension. Top: pre-operative tracings. Observe the figure-of-eight rotation in the horizontal plane and the pattern of incomplete right bundle-branch block. Bottom: post-operative ( 3 months after operation). Note the significant changes that have occurred in the VCG especially in the horizontal plane. 


\section{Acyanotic Congenital Heart Disease}

Atrial Septal Defect-Secundum Type. Frontal plane: the loop in this plane may be normal. The predominant rotation is clockwise with a tendency for the maximum deflection vector to be directed inferiorly and to the right (Hamer, 1958; Liebman and Nadas, 1960; Abramson and Burton, 1962). There is usually a delay in the terminal component of the QRS vector representing some form of right ventricular conduction delay. The terminal component of the loop is usually located in the superior and rightward quadrant.

Sagittal plane: the initial component is normal. The body of the loop is displaced anteriorly though the rotation in most cases continues to be counterclockwise.

Horizontal plane: the initial component is normally directed anteriorly and to the right and has a normal magnitude. The rotation is predominantly clockwise and the main body of the QRS loop is almost entirely located anteriorly. There is a delay in the terminal component of the QRS vector which is located in the anterior or posterior but always in the right quadrant. This type of loop most likely represents right ventricular hypertrophy with right ventricular conduction defect, related to late depolarization of the outflow tract of the right ventricle. This loop is quite distinct from the benign incomplete right bundle-branch block seen in normal subjects by virtue of the fact that in the latter condition the rotation is usually counterclockwise (Fig. 5). These abnormalities often improve after corrective operation for this type of cardiac defect.

If pulmonary hypertension develops in this disorder significant changes in the QRS loop occur in all three planes: there is a greater degree of rightward displacement in the frontal plane, the rotation becomes clockwise in the sagittal plane, and the horizontal loop becomes large and of oval shape

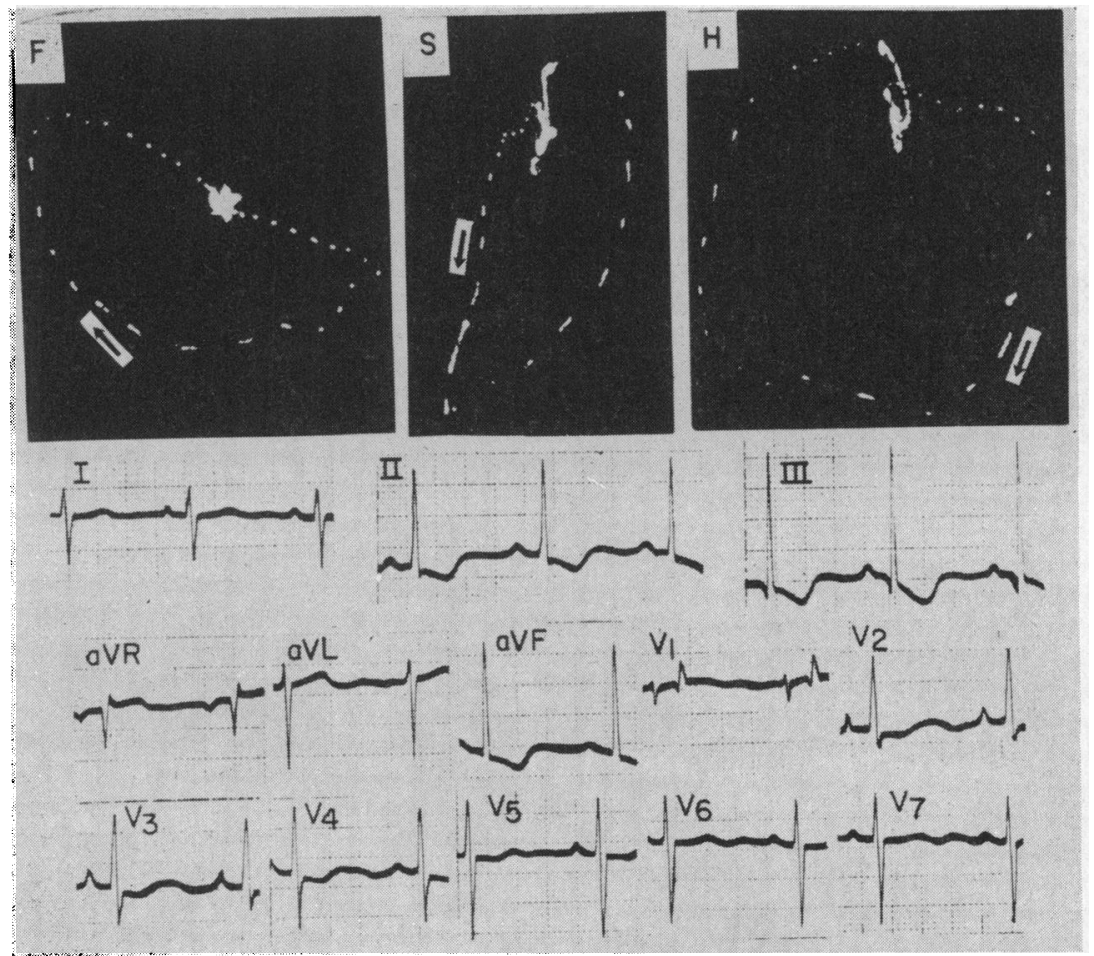

Fig. 6.-Atrial septal defect with pulmonary hypertension. Note the "oval"-shaped loop in the horizontal plane and the clockwise rotation in the frontal and horizontal planes. The loop is very displaced inferiorly, anteriorly, and to the right, indicating the presence of severe right ventricular hypertrophy. Observe the initial vector in the horizontal plane directed posteriorly and to the left. 

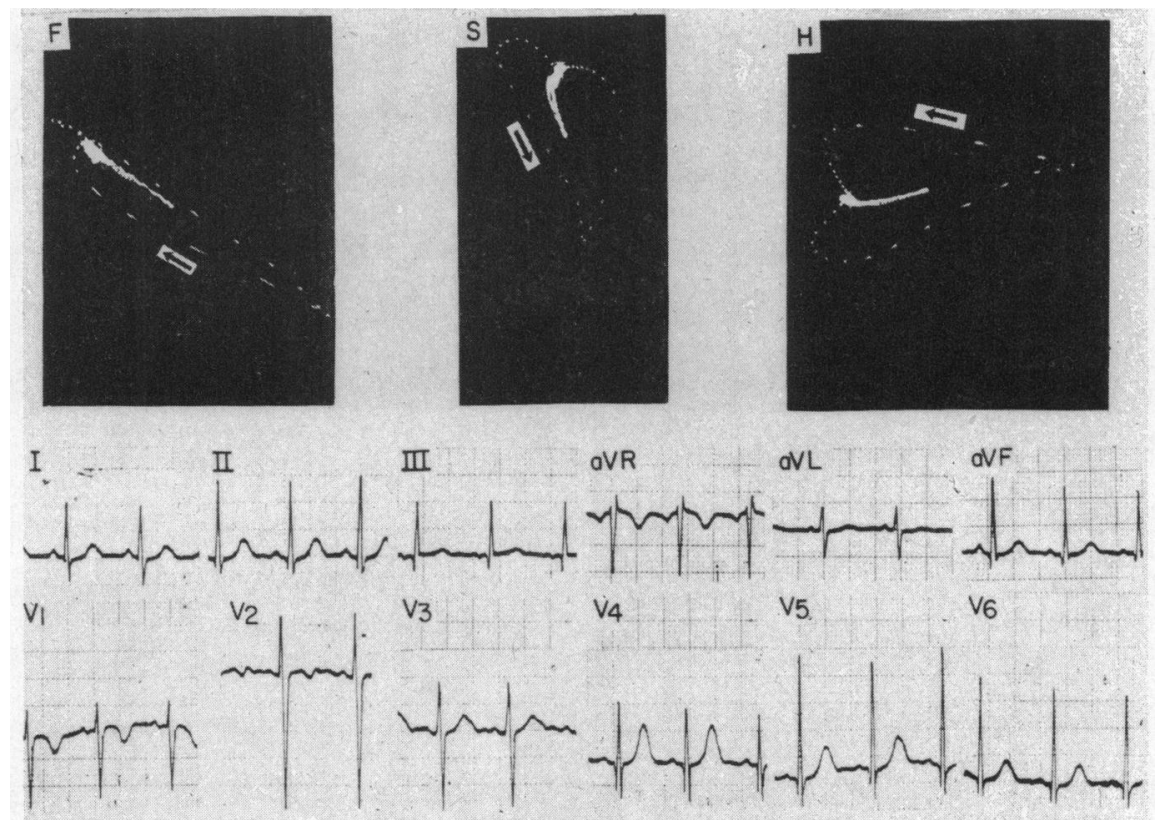

FIG. 7.-Ventricular septal defect. Note counterclockwise rotation in the sagittal and horizontal loops. There is a large septal vector directed anteriorly and to the right, indicating septal hypertrophy.

and very displaced anteriorly and to the right. There is also a change in direction of the initial component which is anterior and to the left. These changes are directly related to the degree of pulmonary hypertension (Fig. 6).

Ventricular Septal Defect. Frontal plane: the QRS loop is usually normal in this plane in cases of uncomplicated ventricular septal defect. The rotation in this plane can be either clockwise or counterclockwise. The maximum deflection vector averages 65 to 90 degrees (Dack, 1960).

Sagittal plane: the rotation is counterclockwise with a maximum deflection vector displaced anteriorly and inferiorly.

Horizontal plane: this is the most useful loop in the diagnosis of ventricular septal defect. The initial septal vector is significantly increased in magnitude and is located in the anterior and right quadrant. The rotation of the loop is clockwise or in figure-of-eight with the efferent limb rotating counterclockwise. The maximum deflection vector is in the posterior and left quadrant though there is a large portion of the loop in the anterior and left quadrant. Of importance is the fact that there is a large terminal component located in the right and anterior quadrant. This type of loop reflects the presence of septal, right, and left ventricular hypertrophy, and the delayed later terminal component probably represents delay in the activation of the hypertrophied outflow tract of the right ventricle. The loop in the horizontal plane is so characteristic that one should suspect the presence of ventricular septal defect when these findings are recorded in this plane (Fig. 7).

If pulmonary hypertension develops in a patient with ventricular septal defect the following changes occur in a fairly predictable way; the frontal loop is displaced inferiorly and to the right with clockwise rotation, the sagittal and horizontal loops rotate clockwise and are located in the anterior quadrant, there is a decrease in the amplitude of the initial septal vector in the horizontal loop, the large T loop is decreased in amplitude and is opposed to the QRS vector indicating increase in the ventricular gradient. These changes represent increase in pulmonary pressure with great loading of the right ventricle and relative decrease in the left ventricular work. Since the signs of 


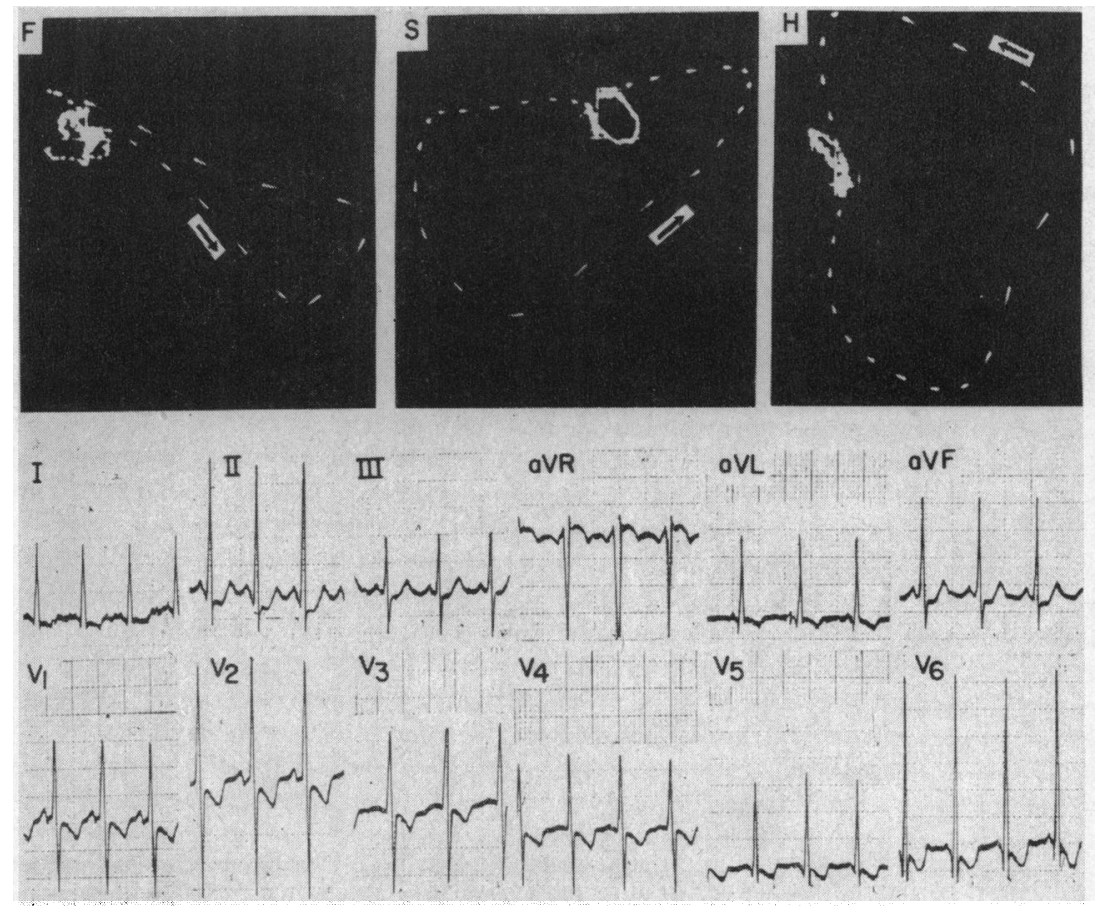

FIG. 8.-Patent ductus arteriosus. Note counterclockwise rotation in all three planes. There is a large septal vector best seen in the horizontal plane. Observe the anterior displacement of the loop in the horizontal and sagittal planes, indicating right ventricular hypertrophy.

left ventricular hypertrophy remain present, this becomes of diagnostic importance in separating ventricular septal defect with pulmonary hypertension from cases of tetralogy of Fallot.

Patent Ductus Arteriosus. Frontal plane: the rotation is usually counterclockwise. The maximum deflection vector has increased magnitude and is displaced superiorly (above the zero degree).

Sagittal plane: the rotation is counterclockwise. The initial component has an increased magnitude and is located in the superior and anterior quadrant.

Horizontal plane: the loop is opened. The initial septal vector has high voltage and is inscribed anteriorly and to the right, the rotation is counterclockwise and the maximum deflection vector is displaced posteriorly around 270 degrees. In contrast to what is found in ventricular septal defect, patients with uncomplicated patent ductus arteriosus showed small electrical forces in the anterior and left quadrant. This is due to lack of hypertrophy of the right ventricular free wall in patients with uncomplicated patent ductus arteriosus (Fig. 8).

If pulmonary hypertension is present there is change in rotation in all 3 planes with the loops rotating clockwise if there is right ventricular preponderance (Luna and Crow, 1961; Cabrera and Gaxiola, 1959, 1960). There is also an increase in magnitude of the maximum deflection vector with a large anterior and rightward displacement of the loop in the sagittal and horizontal planes.

Pulmonary Stenosis, Isolated. The changes in the VCG reflect the degree of severity of the obstruction. All forms of changes in rotation, spatial orientation and displacement, and changes in magnitude are seen.

Frontal plane: in mild cases in which the systolic gradient across the pulmonary valve is small $(10-40 \mathrm{~mm}$. Hg) the frontal loop may be normal in direction, rotation, magnitude, and spatial orientation. This holds true to the sagittal and horizontal loops as well. With increase in the systolic gradient $(40-80 \mathrm{~mm}$. $\mathrm{Hg})$ the frontal loop will rotate clockwise with increased magnitude of the 


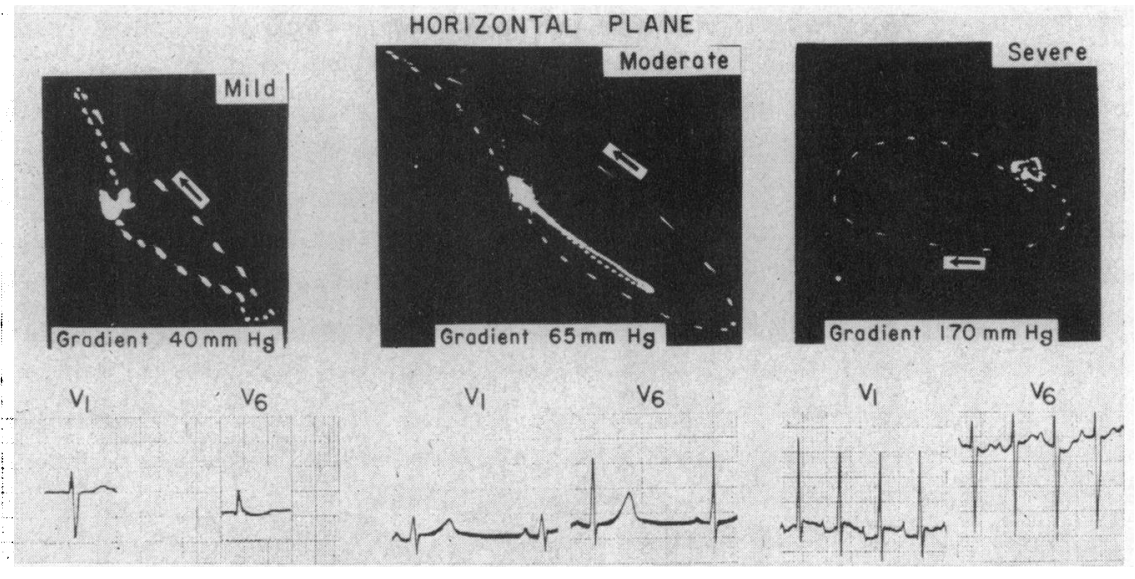

FIG. 9.-Isolated pulmonary stenosis at various degrees of severity. Note a progressive anterior displacement of the QRS loop. Observe clockwise rotation in the case with severe stenosis. Cases of mild to moderate severity are sometimes difficult to differentiate.

maximum deflection vector. The main body of the loop is located in the inferior and rightward quadrant. With a severe degree of stenosis (above $88 \mathrm{~mm}$. $\mathrm{Hg}$ ) the loop will show a marked rightward and superior displacement of the QRS vector.

Sagittal plane: the rotation may be counterclockwise in mild stenosis, becoming always clockwise in moderate and severe cases. The initial septal component has a decreased magnitude or is displaced to the left in severe cases, being normal in mild cases of stenosis. Therefore, the loop starts anteriorly and to the left. There is a large anterior displacement of the QRS vector and the loop passes through a stage of transition in which it will go from a predominant position in the right anterior quadrant to a left anterior quadrant and finally to a right posterior quadrant. These changes reflect the progressive increase in the degree of right ventricular hypertrophy (Fig. 9).

The changes in the $T$ vector occur in the opposite direction to the QRS vector indicating the progressive increase of right ventricular ischæmia.

Vectorcardiograms will not differentiate valvular from infundibular stenosis, regardless of the degree of stenosis.

If a ventricular septal defect is present the initial component in the horizontal plane will show an increased magnitude being located in the anterior and rightward quadrant. This component represents septal hypertrophy as a result of left-to-right shunt (Fig. 10).

Aortic Stenosis, Coarctation of the Aorta, and Idiopathic Myocardial Hypertrophy. These congenital defects exhibit VCG findings of various degrees of left ventricular hypertrophy, these being more conspicuous in cases of aortic stenosis.

Frontal plane: the rotation can be either clockwise or counterclockwise. The loop is usually narrow, and the direction of the maximum deflection vector is usually normal. In a few cases, there may be superior displacement of the QRS vector with counterclockwise rotation. The magnitude of the maximum deflection vector is increased (Braunwald et al., 1955; Wallace, McCall, and Estes, 1962; Hugenholtz, Lees, and Nadas, 1962).

Sagittal plane: the initial component is normal. The rotation is counterclockwise and the main body of the QRS vector is usually located around 70 to 90 degrees.

Horizontal plane: the initial component has diminished amplitude or may be absent. The rotation is always counterclockwise with a significant posterior displacement of the QRS vector. The maximum deflection vector is located around 270 degrees. The $40 \mathrm{msec}$. vector is of useful diagnostic importance and is directed to around 230 degrees. The loop is smooth and narrow. There is anterior displacement of the O-point and therefore the loop does not close completely. 


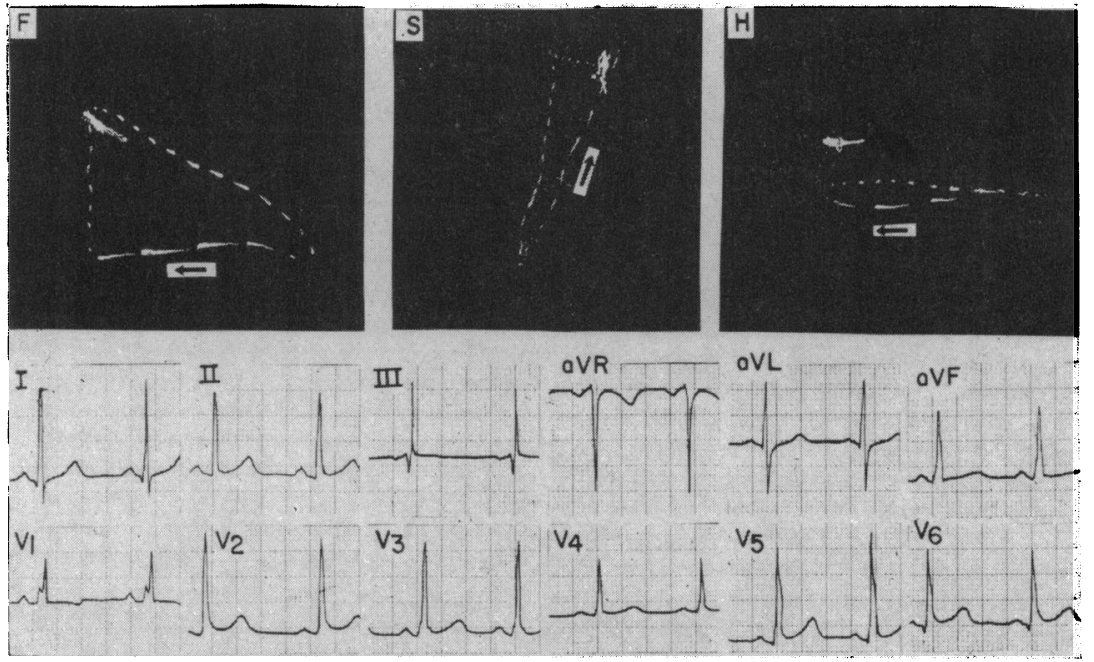

FIG. 10.-Pulmonary stenosis with ventricular septal defect and a left-to-right shunt. Note the anterior and rightward displacement of the QRS vector which is indicative of right ventricular hypertrophy. Observe also the large initial vector in the horizontal loop which suggests the presence of septal hypertrophy.

The $\mathbf{T}$ vector is not enlarged and is displaced anteriorly, indicating increased left ventricular gradient and myocardial ischæmia secondary to the left ventricular hypertrophy (Fig. 11). There is no possibility of differentiating valvular from subvalvular stenosis by the VCG.

Cases with idiopathic myocardial hypertrophy present very interesting vectorcardiographic findings that simulate the picture of myocardial infarction. These changes probably represent areas
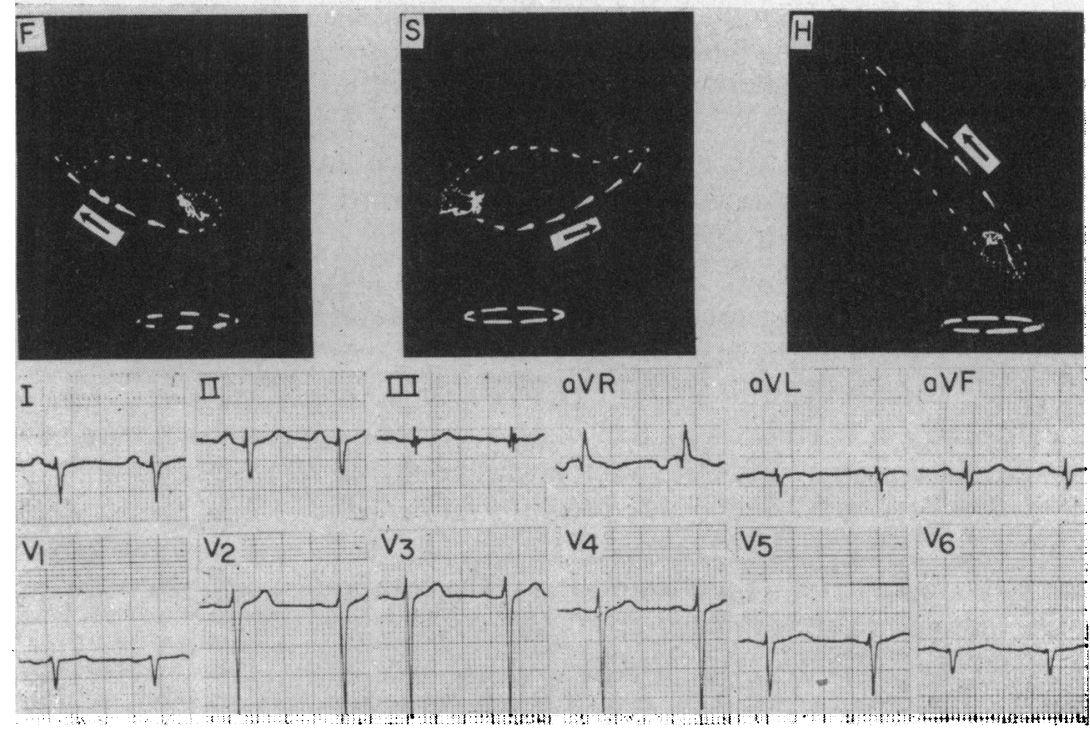

FIG. 11.-Idiopathic myocardial hypertrophy involving the left ventricle without gradient across the aortic valve. Note the marked posterior displacement of the QRS vector in the horizontal plane indicative of left ventricular hypertrophy. 


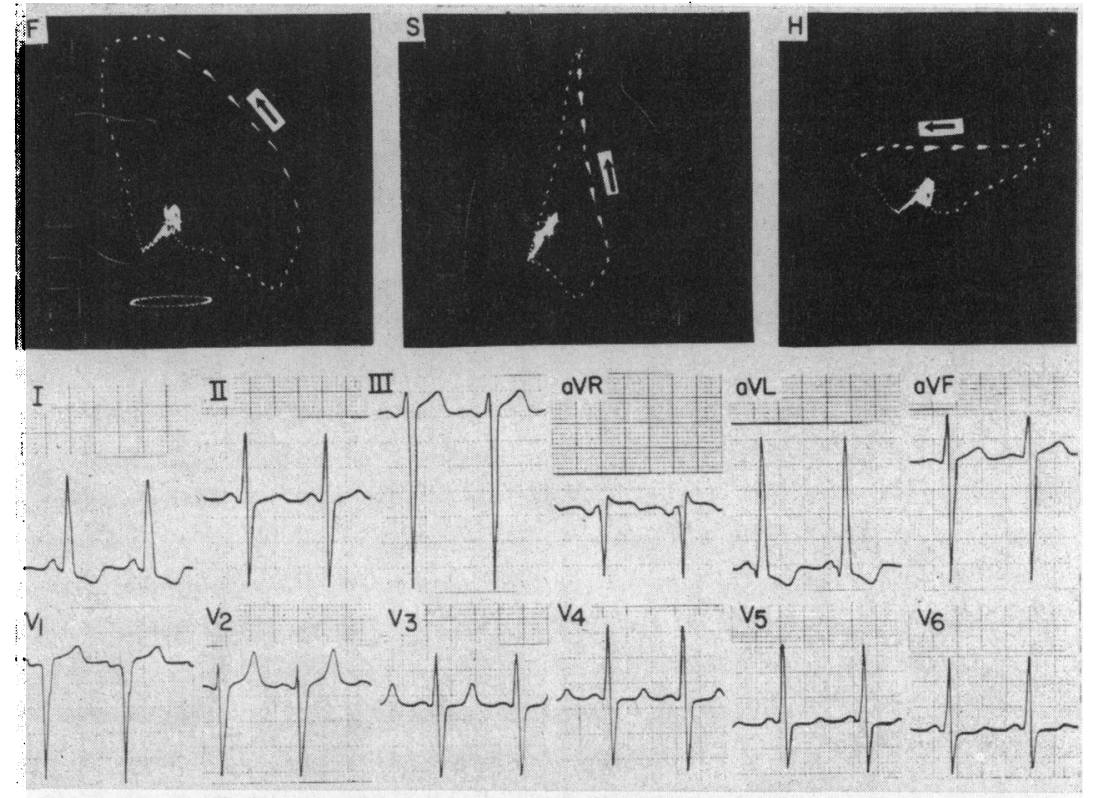

Fig. 12.-Severe sub-aortic stenosis. Note counterclockwise rotation in all three planes. The loop is very displaced superiorly and posteriorly, indicating hypertrophy of the basal surface of the left ventricle. Note the initial component in the horizontal plane directed to the left instead of to the right.

of diffuse myocardial fibrosis which is the end-result of severe myocardial hypertrophy. Though almost all types of infarction can be detected, the most common is the anterior or lateral. These loops are characterized by a lack of anterior force in the horizontal plane with clockwise or counterclockwise rotation (Fig. 12). There may be a large anterior and rightward initial component which, if present, suggests the extension of the infarction pattern to the lateral wall. In these cases, anomalous origin of the coronary arteries should always be suspected and ruled out by left heart catheterization. It is well worth keeping in mind, however, that these patterns described above do occur in cases of idiopathic myocardial hypertrophy in the absence of anomalous coronary arteries.

Isolated involvement of the right ventricle sometimes occurs in this disease and the QRS loop in these cases will have an increased magnitude and will be located in the anterior and rightward quadrant (Fig. 13).

Atrio-ventricular Canal-Ostium Primum. Frontal plane: the rotation is almost always counterclockwise with a marked superior and leftward displacement of the main body of the QRS vector, representing left ventricular overload (Toscano-Barbosa, Brandenburg, and Burchell, 1956; Beregovich et al., 1960). The initial forces are directed inferiorly and to the left but this is mainly dependent on the orientation of the maximum deflection vector.

Sagittal plane: the rotation is counterclockwise with the initial vectors in the anterior and inferior quadrants. The main body of the loop is displaced posteriorly and superiorly with a delay in the terminal component.

Horizontal plane: there is usually a very large septal vector located in the anterior and rightward quadrant. The rotation is counterclockwise and the maximum deflection vector is in the posterior and left or right quadrant. Occasionally there is an anterior displacement of the maximum deflection vector that reflects the degree of hypertrophy of the free wall of the right ventricle. There is also a large terminal component located in the right anterior quadrant with a delayed inscription (Fig. 14).

The $T$ vector is large and inscribed in the same direction as the QRS vector, provided that pulmonary hypertension of significant degree is not present. 


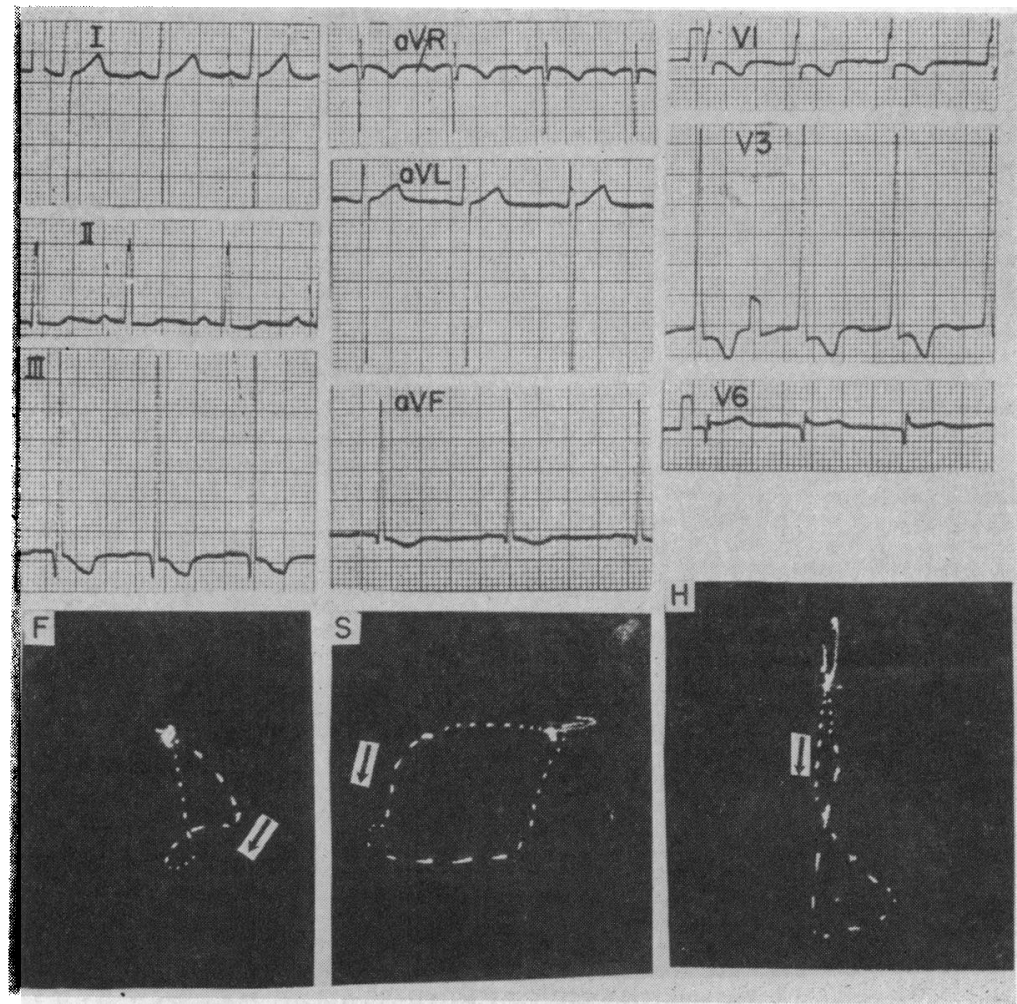

FIG. 13.-Idiopathic myocardial hypertrophy involving the right ventricle. Note extreme degree of anterior displacement of the QRS loop in the sagittal and horizontal planes reflecting severe degree of right ventricular hypertrophy. Observe also the deep $\mathbf{Q}$ waves in V6 in the electrocardiogram, which simulate the presence of lateral wall infarction. This wave represents severe degree of septal hypertrophy.
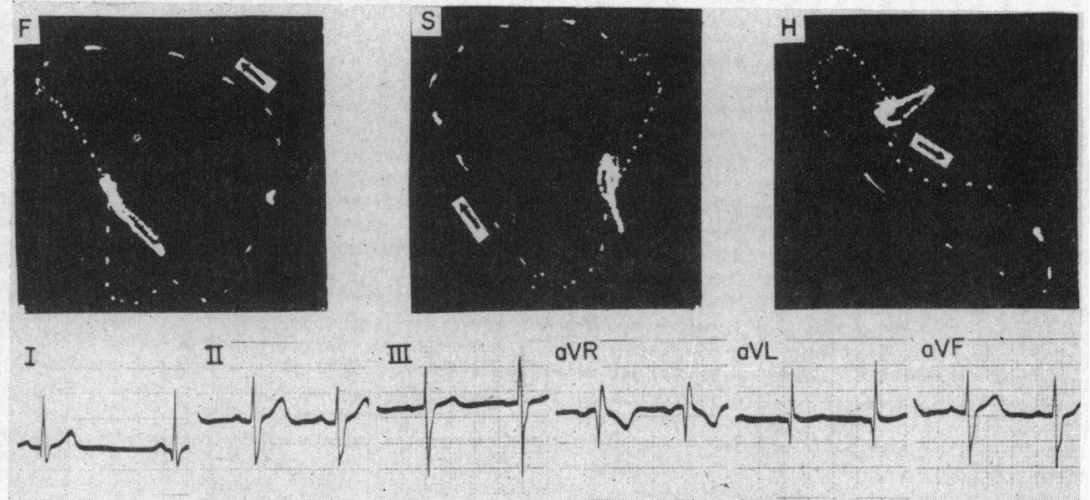

aVL

aVF

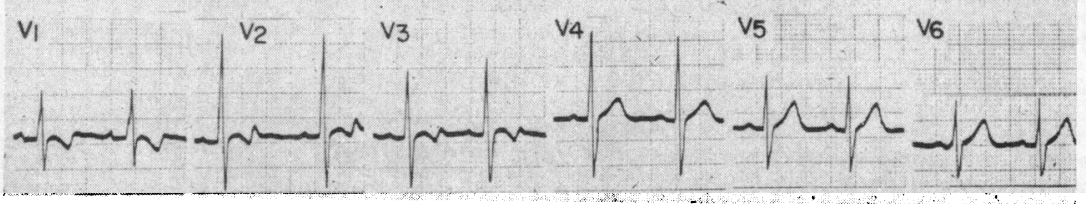

FIG. 14.-Ostium primum. Note the typical superior displacement of the QRS vector with counterclockwise rotation in the frontal plane associated with an anterior displacement and clockwise rotation in the horizontal plane. This type of loop is extremely suggestive of this condition. 


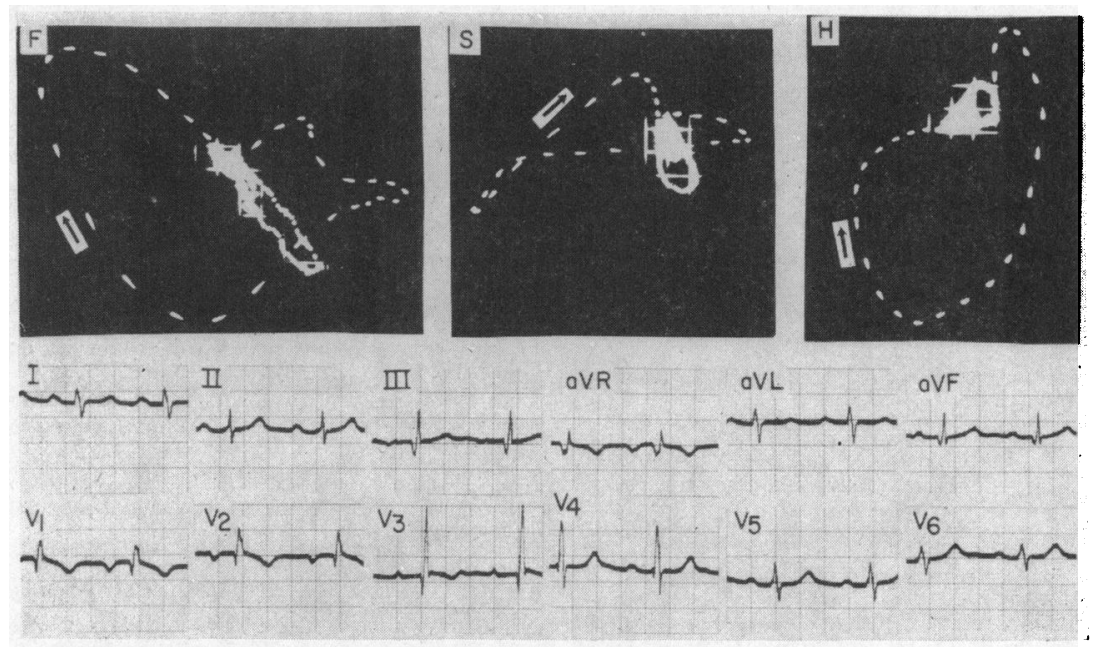

FIG. 15.-Corrected transposition of the great vessels. Note clockwise rotation in all three planes with a large anterior and rightward force. Observe also the initial component directed superiorly, posteriorly, and to the left. The electrocardiogram shows first degree A-V block with a $q R$ pattern in V1.

If pulmonary hypertension takes place the QRS loop will be predominantly situated in the anterior and rightward quadrant. However, an important feature in these cases is the persistence of a large initial vector in the horizontal plane and the superior orientation of the vector in the frontal plane.

Corrected Transposition of the Great Vessels. In this condition there is a reverse of the normal sequence of the ventricular activation.

Frontal plane: a marked degree of left axis deviation is one of the characteristics of this disease. The maximum deflection vector is usually at $\mathbf{3 0 0}$ degrees. The rotation is clockwise with the initial component directed superiorly.

Sagittal plane: the rotation is counterclockwise with the initial component being directed superiorly and posteriorly.

Horizontal plane: the loop is quite characteristic in this plane. The initial vector is directed posteriorly and to the left instead of anteriorly and to the right. This is probably due to the reverse activation of the ventricular septum. There is also a large anterior displacement of the maximum deflection vector (Fig. 15).

\section{Cyanotic Heart Disease}

(a) Trilogy, Tetralogy, and Pentalogy of Fallot. There are no sure ways to differentiate these malformations by the VCG. However, there are minor changes in the P and QRS vectors that sometimes help in separating these conditions.

Since the basic anomaly that determines the work load of both ventricles is the presence of atrial or ventricular septal defect and pulmonary stenosis, the degree of severity of ventricular loading is primarily based on the degree of stenosis and on the size of the shunt.

Frontal plane: the rotation is always clockwise in this plane. One may suspect the presence of associated left ventricular overload if the loop rotates counterclockwise and as a result the diagnosis of trilogy or pentalogy of Fallot is favoured. There is displacement of the QRS loop inferiorly and to the right (Sodi-Pallares et al., 1958). The maximum deflection vector averages 100 to 120 degrees and this is a useful differentiation between tetralogy and trilogy of Fallot. In the latter condition, the maximum deflection vector is usually displaced farther superiorly and to the right, being around 180 to 200 degrees. The diagnosis of pulmonary atresia or pseudotruncus arteriosus 

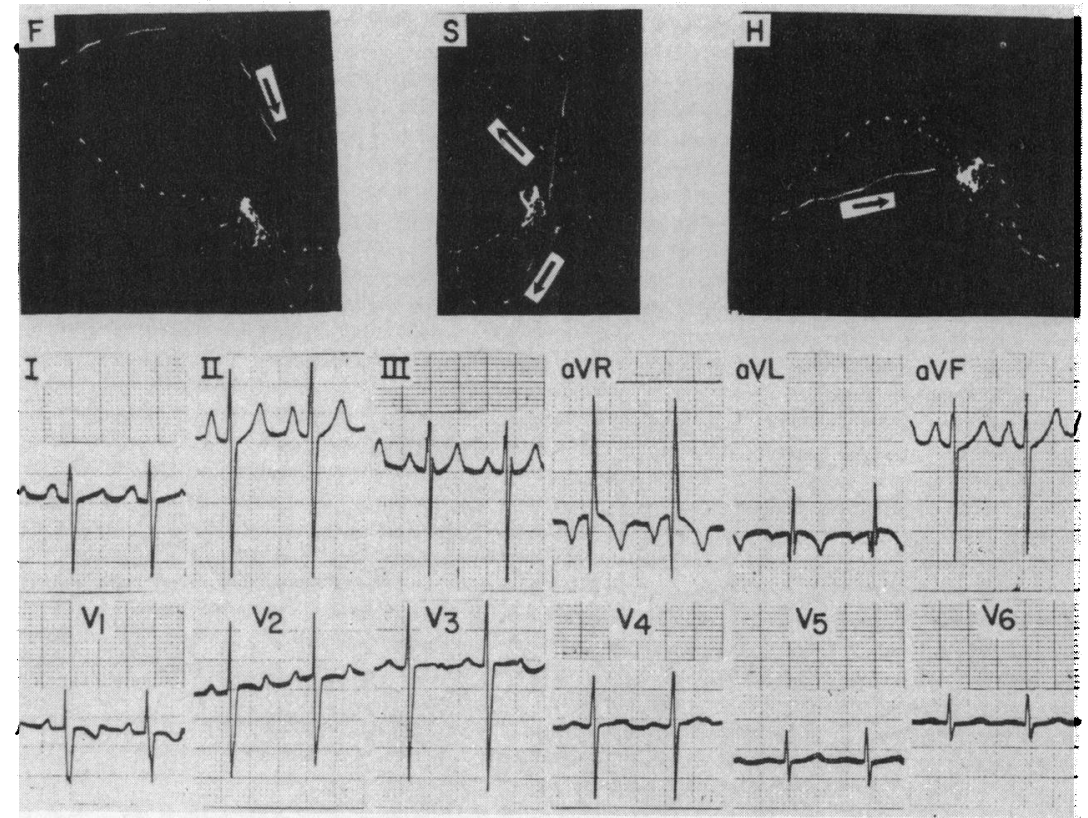

FIG. 16.-Tetralogy of Fallot. Observe the superior, anterior, and rightward displacement of the QRS vector, indicating right ventricular hypertrophy.
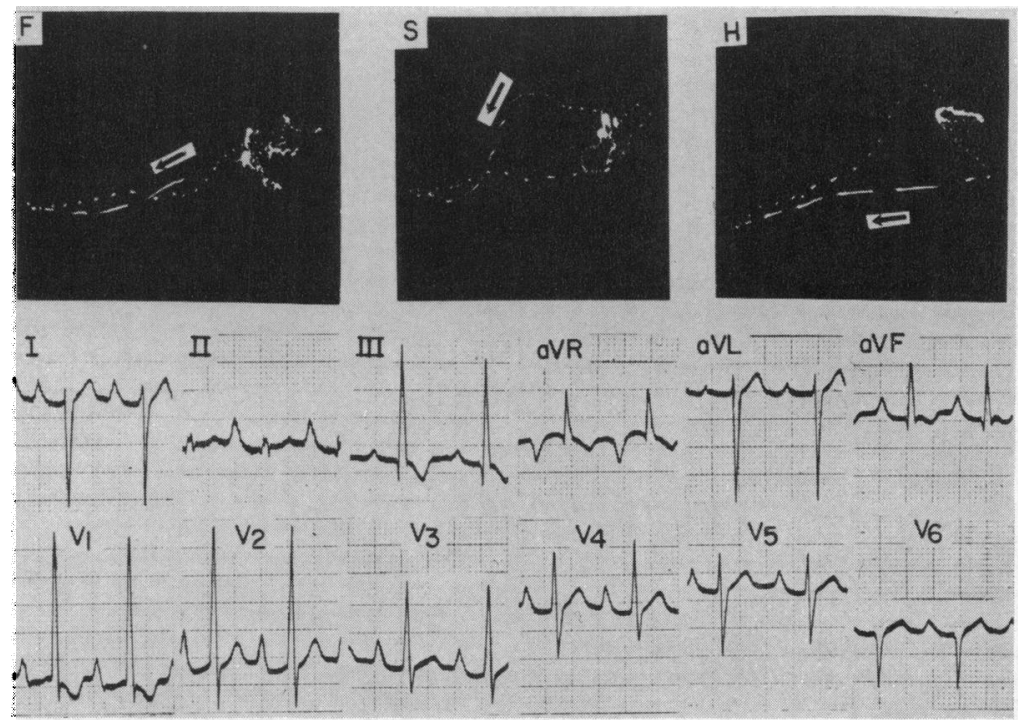

FIG. 17.- Transposition of the great vessels. Note figure-of-eight rotation in the frontal and sagittal planes with clockwise rotation in the horizontal plane. There is anterior and rightward displacement of the loop, indicating right ventricular hypertrophy. The initial counterclockwise rotation in the sagittal plane suggests the presence of associated left ventricular hypertrophy.

should be suspected when there is much superior and rightward displacement of the QRS vector and in this situation the diagnosis of trilogy of Fallot cannot be excluded.

Sagittal plane: the loop is smooth, round, and has increased magnitude. There is always clockwise rotation with anterior and inferior displacement of the maximum deflection vector. 


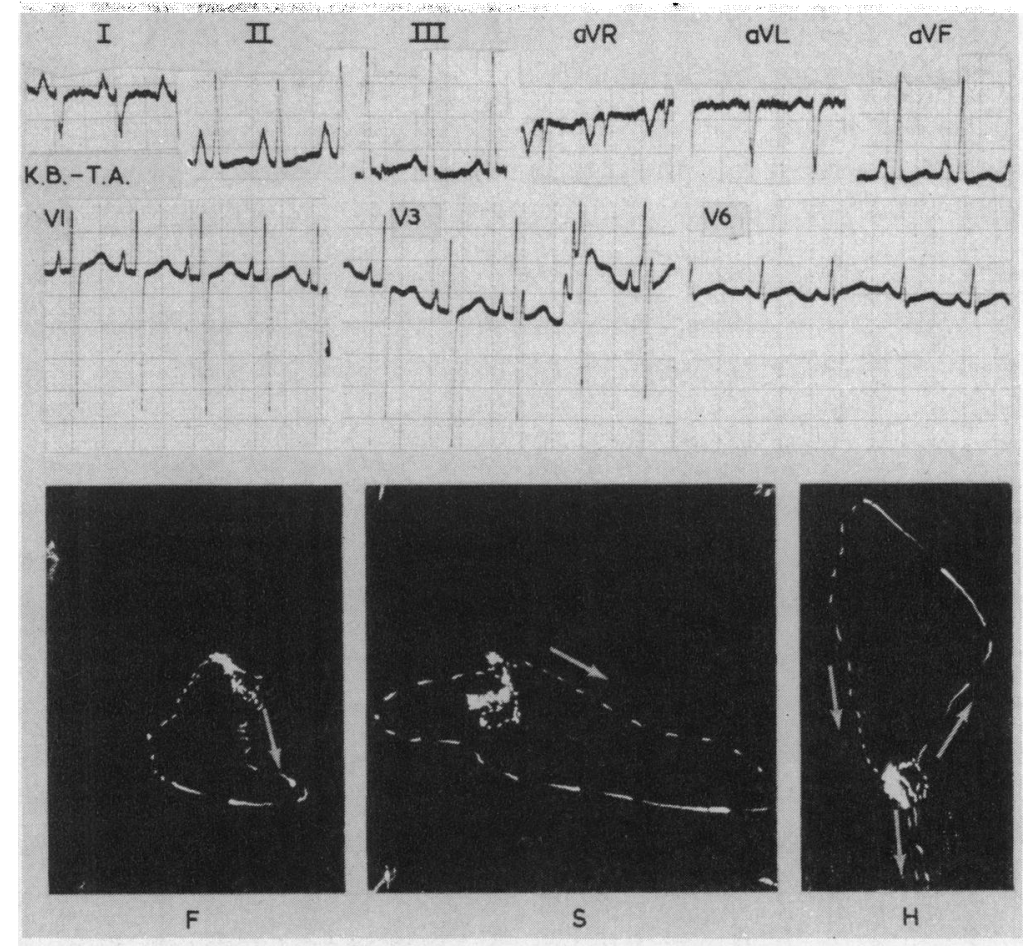

FIG. 18.-Tricuspid atresia. Note the presence of clockwise rotation in the frontal plane with the unusual position of the maximum deflection vector in the inferior quadrant. There is a posterior displacement of the QRS vector in the sagittal and horizontal planes.

Horizontal plane: the rotation is clockwise. The initial septal component is small or absent. The loop is large and oval with the maximum deflection vector being displaced anteriorly and to the right (Pileggi et al., 1962). There is sometimes a terminal component directed posteriorly and to the right. The $\mathrm{O}$-point is displaced and the $\mathrm{T}$ vector is inscribed in the opposite direction to that of the QRS vector. These changes indicate the presence of severe right ventricular hypertrophy. If signs of right ventricular hypertrophy of the degree described above are present and the initial septal vector has an increased magnitude, then the diagnosis of pentalogy of Fallot may be suspected. This occurs as a result of overloading of the left ventricle from a right-to-left shunt at the atrial level (Fig. 16).

Transposition of the Great Vessels. In this condition there is a severe degree of right ventricular hypertrophy, because the right ventricle is maintaining the high pressure systemic circulation. There is also associated left ventricular hypertrophy because of the right-to-left shunt at the atrial level.

Frontal plane: the rotation is usually clockwise. The maximum deflection vector has an increased magnitude and is directed inferiorly and to the left. Extreme degree of right axis deviation of the QRS vector is unusual because of the associated presence of left ventricular hypertrophy which tends to balance the electrical preponderance of the hypertrophied right ventricle. Thus, left axis deviation is sometimes seen in this condition and specially seen in cases with associated atrial and ventricular septal defect (Shaher, 1963).

Sagittal plane: the rotation is clockwise with anterior and inferior displacement of the QRS vector.

Horizontal plane: the initial septal vector is preserved. The rotation is clockwise and the major 
portion of the loop is located in the anterior and left quadrant. There is practically no posterior force (Fig. 17).

Truncus Arteriosus. The findings in truncus arteriosus do not differ significantly from the ones described for tetralogy of Fallot.

Tricuspid Atresia. Frontal plane: the rotation is counterclockwise. The maximum deflection vector is directed superiorly and to the left. In a few cases there is an inferior displacement of the loop (Fig. 18).

Sagittal and horizontal planes: the rotation is counterclockwise with a posterior and leftward displacement of the QRS vector. These changes reflect the presence of left ventricular hypertrophy with electrical forces which are unopposed by the hypoplastic right ventricle.

\section{Discussion}

Vectorcardiography is a useful adjunct to electrocardiography in the diagnosis of congenital heart disease. In a few instances it proves to be a superior technique since it provides instantaneous recording of individual vectors in two axes that cannot be done with the use of the electrocardiogram. Vectorcardiographic findings do have a good correlation with the hæmodynamic findings and, therefore, serve as a useful guide of the work load conditions of the ventricles. This can be demonstrated by the changes that occur in cases with pulmonary stenosis at various degrees of severity, in which conditions the VCG detects with an acceptable degree of accuracy the systolic gradient across the pulmonary valve (Fig. 9). Furthermore, in cases of septal defect with large shunts the VCG appears to have a good correlation with the degree of shunt and severity of pulmonary hypertension as exemplified in cases of ventricular or atrial septal defect (Fig. 5 and 6). The VCG appears to have a distinct advantage over the ECG in the presence of incomplete right bundlebranch block. Differentiation of uncomplicated right bundle-branch block in most instances from right bundle-branch block as occurs in cases of atrial septal defect can be made on the basis of rotation of the loop in the horizontal plane.

Since both techniques are recording the same electrical phenomenon, the reason for superiority of one in relation to another is difficult to understand. Though these discrepancies do occur in a few instances, in the majority of cases the ECG provides the same information recorded in the VCG. The superiority of the VCG in these few instances appears to be related to placement of the electrodes in the chest wall, to the balance of resistances in the electrical circuitry, and to the twodimensional visualization of the electrical events recorded in the VCG. The better displacement of the electrical events in the VCG makes this technique very valuable in the diagnosis of various forms of congenital and acquired heart diseases.

\section{SUMMARY}

A review of the usefulness of vectorcardiography in the diagnosis of congenital heart disease is made. The vectorcardiograms were recorded with the use of the Frank system of electrode placement.

Vectorcardiograms reflect with a great degree of accuracy the hæmodynamic events of the right and left heart in cases of pressure and/or volume loading.

The analysis of the QRS loops provides quick, accurate, and reliable information of the various vectors of ventricular activation. Cases with septal, free wall, and outflow tract hypertrophy can be separated by this technique in the majority of cases.

Vectorcardiography is a useful adjunct to electrocardiography in the diagnosis of cyanotic and acyanotic congenital diseases.

We are grateful to Mrs. Mary Vensel, Miss Shirley Cardwell, and Miss Els Boesten for their technical assistance. We also wish to thank Dr. Francis Giknis, Chief of Cardiology of the San Diego Naval Hospital, for permission to study a few of the cases that form the basis of this study. 


\section{REFERENCES}

Abramson, H., and Burton, C. R. (1962). The spatial vectorcardiogram in proven atrial septal defect. Brit. Heart J., 24, 103.

Beregovich, J., Bleifer, S., Donoso, E., and Grishman, A. (1960). The vectorcardiogram and electrocardiogram in persistent common atrioventricular canal. Circulation, 21, 63.

Braunwald, E., Sapin, S. O., Donoso, E., and Grishman, A. (1955). A study of the electrocardiogram and vectorcardiogram in congenital heart disease. III. Electrocardiographic and vectorcardiographic findings in various malformations. Amer. Heart J., 50, 823.

Bristow, J. D. (1961). A study of the normal Frank vectorcardiogram. Amer. Heart J., 61, 242.

Burch, G. E., Abildskov, J. A., and Cronvich, J. A. (1953). Spatial Vectorcardiography. Lea and Febiger, Philadelphia.

Burger, H. C., Van Milaan, J. B., and Boer, W. den (1952). Comparison of different systems of vectorcardiography. Brit. Heart J., 14, 401.

Cabrera, E., and Gaxiola, A. (1959). A critical re-evaluation of systolic and diastolic overloading patterns. Progr. cardiovasc. Dis., 2, 219.

- and - (1960). Diagnostic contribution of the vectorcardiogram in hemodynamic overloading of the heart. Amer. Heart J., 60, 296.

Dack, S. (1960). The electrocardiogram and vectorcardiogram in ventricular septal defect. Amer. J. Cardiol., $5,199$.

Duchosal, P. W., and Sulzer, R. (1949). La Vectorcardiographie (Bibliotheca cardiologica Fasc 3). Karger, Basel.

Forkner, C. E., Hugenholtz, P. G., and Levine, H. D. (1961). The vectorcardiogram in normal young adults. Frank lead system. Amer. Heart J., 62, 237.

Frank, E. (1956). An accurate, clinically practical system for spatial vectorcardiography. Circulation, $13,737$.

Grishman, A., Borun, E. R., and Jaffe, H. L. (1951). Spatial vectorcardiography: Technique for the simultaneous recording of the frontal, sagittal, and horizontal projections. Amer. Heart J., 41, 483.

Hamer, N. A. (1958). The vectorcardiogram in atrial septal defect. Brit. Heart J., 20, 215.

Helm, R. A. (1956). Vectorcardiographic notation. Circulation, 13, 581.

Hugenholtz, P. G., Lees, M. M., and Nadas, A. S. (1962). The scalar electrocardiogram, vectorcardiogram, and exercise electrocardiogram in the assessment of congenital aortic stenosis. Circulation, 26, 79.

—- and Liebman, J. (1962). The orthogonal vectorcardiogram in 100 normal children (Frank system). Circulation, 26, 891.

Jouve, A., Buisson, P., Albouy, A., Velasque, P., and Bergier, G. (1950). La Vectorcardiographie en Clinique, p. 127. Masson, Paris.

Langner, P. H., Okada, R. H., Moore, S. R., and Fies, H. L. (1958). Comparison of four orthogonal systems to vectorcardiography. Circulation, 17, 46.

Liebman, J., and Nadas, A. S. (1960). The vectorcardiogram in the differential diagnosis of atrial septal defect in children. Circulation, 22, 956.

Luna, R. L., and Crow, E. W. (1961). Correlation of degree of pulmonary hypertension with morphology of the QRS lead in $\mathrm{V}_{1}$ in cases with evidence of systolic overloading of the right ventricle. Amer. Heart J., 62,481 .

McCall, B. W., Wallace, A. G., and Estes, E. H. (1962). Characteristics of the normal vectorcardiogram recorded with the Frank lead system. Amer. J. Cardiol., 10, 514.

McFee, R., and Johnston, F. D. (1954). Electrocardiographic leads. III. Synthesis. Circulation, 9, 868.

Pileggi, F., Ebaid, M., Tranchesi, T., Macruz, R., and Décourt, L. V. (1962). The vectorcardiogram in ventricular septal defect associated with pulmonary stenosis. A study of 60 cases. Amer. Heart J., $63,25$.

Pipberger, H. V. (1959). Current status and persistent problems of electrode placement and lead systems for vectorcardiography and electrocardiography. Progr. cardiovasc. Dis., 2, 248.

Med., 25, 539.

Scherlis, L., Lasser, R. P., and Grishman, A. (1951). Spatial vectorcardiography. The normal vectorcardiogram. Amer. Heart J., 42, 235.

Schmitt, O. H., and Simonson, E. (1955). The present status of vectorcardiography. Arch. intern. Med., $96,574$.

Shaher, R. M. (1963). Left ventricular preponderance and left axis deviation in cyanotic congenital heart disease. Brit. Heart J., 25, 726.

Simonson, E., Schmitt, O. H., and Levine, R. B. (1955). Comparison of SVEC-measured instantaneous spatial vectors, with mean vectors derived from conventional ECG leads. Fed. Proc., 14, 139.

Sodi-Pallares, D., Pileggi, F., Cisneros, F., Ginefra, P., Portillo, B., Medrano, G. A., and Bisteni, A. (1958). The mean manifest electrical axis of the ventricular activation process (ÂQRS) in congenital heart disease: A new approach in electrocardiographic diagnosis. Amer. Heart J., 55, 681.

Toscano-Barbosa, E., Brandenburg, R. O., and Burchell, H. B. (1956). Electrocardiographic studies of cases with intracardiac malformations of the atrioventricular canal. Proc. Mayo Clin., 31, 513.

Wallace, A. G., McCall, B. W., and Estes, E. H. (1962). The vectorcardiogram in left ventricular hypertrophy: a study using the Frank lead system. Amer. Heart J., 63, 466.

Wilson, F. N., and Johnston, F. D. (1938). The vectorcardiogram. Amer. Heart J., $16,14$. 